\title{
Traditional chinese medical nutrition and western nutrition
}

\author{
Volume 4 Issue 2 - 2017 \\ Youya Gao \\ Department of Chemistry, DePaul University, USA
}

\begin{abstract}
Correspondence: Youya Gao, Department of Chemistry, DePaul University, I I I 0 West Belden Avenue, Chicago, USA, Tel 8472201493,Email youyagao@gmail.com
\end{abstract}

Received: February 13, 2017| Published: February 21, 2017
Keywords: traditional chinese medical nutrition, western nutrition, yin-yang, five-elements, epidemiological, proteins, carbohydrates, fats, vitamins, minerals, dietary, recommendations, rye, buckwheat, quinoa

\section{Introduction}

Although both Western and Chinese nutrition share the similar belief that proper nutrition is essential for achieving optimal health, their view about nutrition is different. Western nutritionists study food compositions - proteins, carbohydrates, fats, vitamins, and minerals and make dietary recommendations based on scientific experiments and epidemiological studies. Such as My Plate icon from the new United States Department of Agriculture (USDA), the key nutritional messages of the 2010 Dietary Guidelines for Americans are "balance, variety, moderation and adequacy." In addition, the progress of Western nutrition towards personalized diets based on one's genetic components.

While in the language of traditional Chinese medicine (TCM), food is considered as both nutrients and medicine for the purpose of achieving balance and harmony within the body. The expression of Yin-Yang and Five-Elements characterize both the person and the food. Yin-Yang allows for the description between two extremes - hot and cold, the Five-Elements give the further illustration by providing shape, character, and hue. Furthermore, foods are selected to correspond to an individual's pattern and modified based on other important factors including lifestyle, environment, climate, and season. Together, these properties determine which foods are the most beneficial for each individual.

\section{Yin-yang theory in traditional chinese medical (TCM) nutrition}

The theory of yin and yang is the most fundamental concept of traditional Chinese medicine. One of the major beliefs of TCM is that everything in the universe is either yin or yang, they combine in a complementary manner. Usually, yang is associated with functional aspect of an object and has more energetic qualities such as hot, ascending, bright. Yin, on the other hand, is associated with an object that has less energetic qualities such as cold, descending, dark. However, nothing is totally yin or totally yang, but a balance between the two forces.

The yin-yang symbol consists of a circle, divided by a curved line into a black (yin) and white (yang) side. The curve symbolizes the constant change of balance between yin and yang. Each side contains a small circle of the opposite color. This demonstrates the belief that nothing is never really all yin or all yang. The symbol is as Figure 1.

Viewing the body as a whole, all the portions and organs can be generalized and explained by the yin-yang relationship. And the sum total of yin and yang will be in balance. The following are the examples of yin-yang pairs in the body ${ }^{1}$ (Table 1):

Table I Examples of yin-yang pairs in the body

\begin{tabular}{ll}
\hline Yin & Yang \\
\hline Interior & Exterior \\
Front & Back \\
Body & Head \\
Below the waist & Above the waist \\
Blood and body fluids & Energy (Qi) \\
Liver & Gallbladder \\
Heart & Small Intestine \\
Spleen & Stomach \\
Lung & Large Intestine \\
Kidney & Bladder \\
\hline
\end{tabular}

"Hot and cold" are two terms in which characterizing the properties of food with yin-yang theory. Note that these terms do not literally signify the physical temperature. Instead, they denote the effects of the food on body functions: hot foods promote the generation of heat within the body while cold foods stimulate the release of heat from the body.

a. Examples of hot foods: Rye, buckwheat, quinoa, millet, oat, celery, alfalfa, turnips, radish, bean sprout, cabbage, red bean, kelp, seaweed, bamboo shoot, asparagus, garlic, beet, eggplant.

b. Examples of cold foods: Watermelon, cantaloupe, walnut, sesame, figs, peas, grapes, pineapple, pomegranate, black bean, seafood, mint, chrysanthemum tea, chamomile tea, barley, cucumber, bitter melon, aloe, oyster, clam, basil.

\section{Five elements theory in traditional chinese medical (TCM) nutrition}

Like Yin-Yang theory, Five Elements theory is another important system within traditional Chinese medicine. These five elements are 
a comprehensive template that organizes all natural phenomena into five master groups or patterns in nature. The five groups-Wood, Fire, Earth, Metal, and Water (Figure 2), reflecting a deep understanding of natural law, the universal order underlying all things in our world.

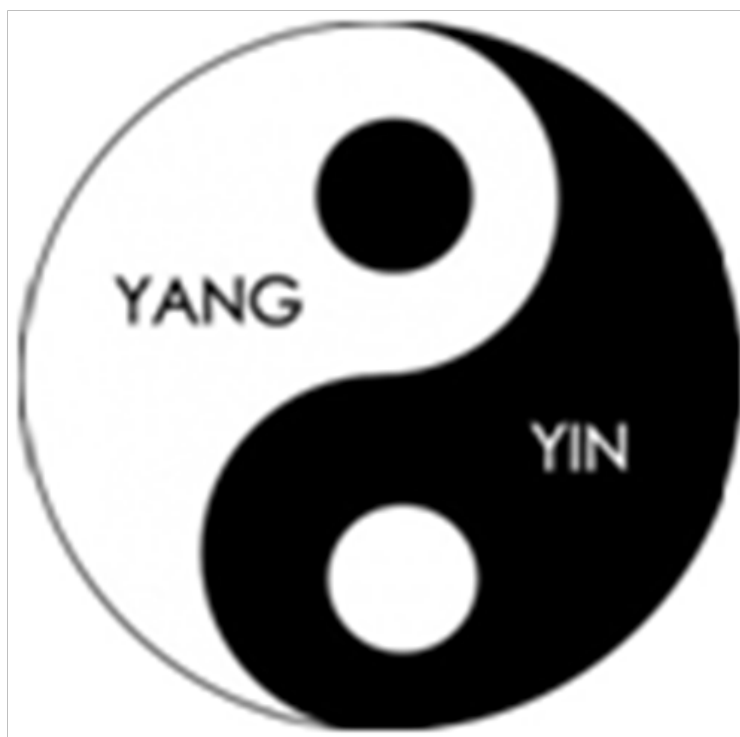

Figure I Symbol of yin-yang.

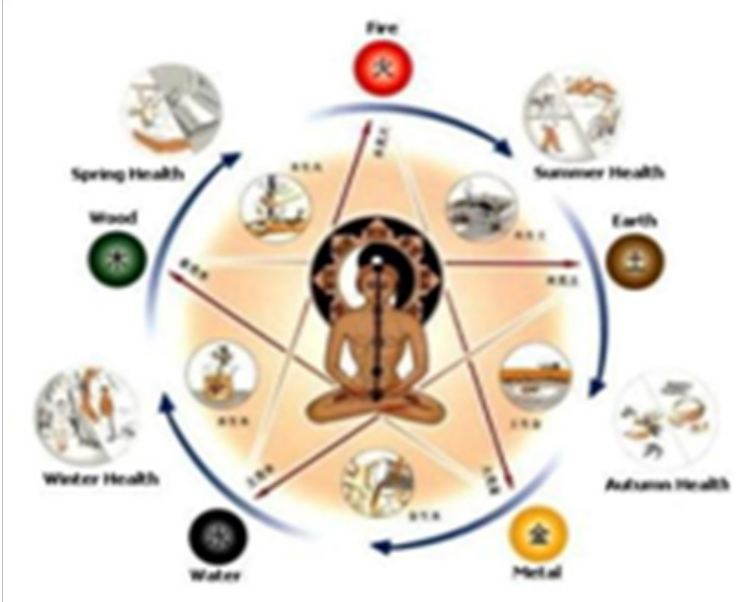

Figure $\mathbf{2}$ Theory of five elements in traditional chinese medicine.

It has been used as a method of diagnosis and treatment for more than 2,000 years, and to associate signs or symptoms to particular organs and afflictions. It explains the processes that occur in the body

Table 2 The processes that occur in the body throughout various stages

\begin{tabular}{llllll}
\hline & Wood & Fire & Earth & Metal & Water \\
\hline Seasons & Spring & Summer & Late Summer & Autumn & Winter \\
Yin Organs & Liver & Heart & Spleen & Lung & Kidney \\
Yang Organs & Gallbladder & Small Intestine & Stomach & Large Intestine & Bladder \\
Tastes & Sour & Bitter & Sweet & Pungent & Salty \\
Tissues & Tendons & Vessels & Muscles & Skin/hair & Bones \\
Colors & Green & Red & Yellow & White & Blue/Black \\
Sense Organs & Eye & Tongue & Mouth & Nose & Ear \\
\hline
\end{tabular}

\section{Conclusion}

While the Western nutrition is scientifically based, traditional Chinese medical nutrition has evolved over thousands of years upon weight loss medications. ${ }^{2,3}$ their favorite ways to achieve optimal health. throughout various stages. Below are the five elements and their corresponding organs, colors, tastes, etc (Table 2).

There are four main cycles within the elements, and interacting with their associated things. The first of these is the generating cycle. In this cycle, each element serves as a "mother," which promotes the growth and development of the following "child" element. Each element provides a generating force or foundation for the element that immediately follows it. For example, the Fire element provides a foundation for the Earth element, and the Earth element provides a nurturing foundation for the Metal element, and so on.

The second main cycle is called controlling cycle. Each element is involved in a check-and-balance relationship that helps keep things in order, and each element both controls and is controlled by another element (Water, for example, controls Fire, but is itself controlled by Earth).

The third and fourth are cycles of imbalance-overactive cycle. An element exerts too much control, over its subordinate element, leading to element damage and imbalances in the body. For example, the Water element may completely put out the Fire element, or the Earth element may soak up the Water element completely.

\section{Two languages of in western and TCM nutrition: obesity}

In the Western nutrition, obesity is considered as excess fat within the body and tissues. There could be many reasons, which may include genetics, diet habit and environment. The main goal for western treatment for obesity is eating fewer calories and having physical exercise. In extreme cases there could be surgery and also

In the TCM area, obesity was first mentioned in Chinese medicine in the Yellow Emperor's Internal Classic of Medicine. The main factors that cause obesity in Chinese medicine are phlegm and dampness. The spleen is responsible for transportation and transformation. When there is a dysfunction of the spleen, there will be an accumulation of dampness and phlegm causing weight gain. There are two different treatments for obesity in TCM. One is herbal treatment. The plan will be based upon each individual's constitution and differentiation of syndromes. The main focus is to transform dampness and phlegm, and facilitate the free flow of qi (energy) and blood in the body. Another treatment is acupuncture, which is a way to unblock qi (energy) and help it flow back into balance by putting very thin needles into your skin at anatomical points. The principal for weight control is that acupuncture not only promotes the flow of (qi) energy but also regulate hormones levels such as insulin, leptin, ghrelin and Cholecystokinin (CCK). ${ }^{4}$

the basis of empirical evidence. Nevertheless, neither system is perfect but instead both have much to learn from the other, blending together to make nutrition global. Allowing all the individuals find 


\section{Acknowledgments}

None.

\section{Conflicts of interest}

Author declares there are no conflicts of interest.

\section{Funding}

None.

\section{References}

1. Paul Pitchford. Healing with Whole Foods: Asian Traditions and Modern Nutrition. (3rd edn), Cookbooks, Food \& Wine, New Jersey, USA. 2002.
2. Kastner J. Chinese Nutrition Therapy: Dietetics in Traditional Chinese Medicine (TCM). (2nd edn), Medicine \& Health Sciences, New Jersey, USA. 2004

3. Cabýoglu MT, Ergene N, Tan U. Treatment of obesity by acupuncture. Am J Clin Nutr. 1976;29(8):832-835.

4. Güçel F, Bahar B, Demirtas C, et al. Influence of acupuncture on leptin, ghrelin, insulin and cholecystokinin in obese women: a randomised, sham-controlled preliminary trial. Acupunct Med. 2012;30(3):203-207. 